\title{
Percepções de acadêmicos de medicina sobre o Regime Letivo Remoto (RLR) durante a pandemia de COVID-19
}

\author{
Medical students' perceptions of the Remote Teaching Regime (RTR) during the COVID- \\ 19 pandemic
}

Percepciones de los estudiantes de medicina acerca del Régimen de Enseñanza Remota (RER) durante la pandemia COVID-19

Mirella Marques Freire ${ }^{1}$, Victória Cristine Araujo Dias ${ }^{1 *}$, Ana Carolina Mathias Santa Rita ${ }^{1}$, Luísa Figueiredo Moreira Gomes ${ }^{2}$, Débora Ignácio Gagossian ${ }^{3}$, Alice Brant Costa ${ }^{1}$, Thiago Luiz do Nascimento Lazaroni'.

\section{RESUMO}

Objetivo: Avaliar a experiência dos graduandos de medicina no Regime Letivo Remoto (RLR) e sua utilização pós pandemia. Métodos: Trata-se de estudo qualitativo relatando a experiência dos acadêmicos de medicina de uma universidade de Minas Gerais durante o RLR. Os estudantes foram convidados virtualmente e receberam o questionário e o Termo de Compromisso Livre e Esclarecido (TCLE) por e-mail. Resultados: Responderam ao questionário $20 \%$ dos estudantes do curso de medicina, predominando mulheres, entre 17 e 26 anos que cursam o ciclo clínico. O RLR impactou negativamente os estudos de $78,1 \%$ dos respondentes e os sentimentos de ansiedade (74,8\%) e desestímulo (73,5\%) predominaram. Mais de $90 \%$ dos estudantes sentem falta das aulas presenciais, da interação social (48\%) e do ambiente acadêmico (45\%). Pontos positivos também surgiram, referentes a rotina de estudos (35,5\%), a ausência de deslocamento (31\%) e utilização dessas ferramentas para algumas atividades pós-pandemia $(40 \%)$. Conclusão: O uso do RLR poderia complementar o ensino médico presencial pós-pandemia. Mais estudos devem ser realizados para entender a realidade do país no RLR e para avanços nas discussões sobre tecnologias alternativas ao ensino médico presencial.

Palavras-chave: Ensino médico, COVID-19, Educação à distância.

\begin{abstract}
Objective: To evaluate the experience of medical students during the Remote Teaching Regime (RTR) and its post-pandemic use. Methods: This is a qualitative study reporting the experience of medical students at a university in Minas Gerais during the RTR. Students were invited virtually and received the questionnaire and signed an informed consent. Results: $20 \%$ of medical students answered the questionnaire, predominantly women, aged between 17 and 26 years, who were attending the clinical part of the course. The RTR negatively impacted the study of $78.1 \%$ of respondents and feelings of anxiety $(74.8 \%)$ and discouragement $(73.5 \%)$ predominated. More than $90 \%$ of the students miss the classroom, social interaction (48\%) and the academic environment (45\%). Positive aspects also highlighted, referring to a better study routine (35.5\%), the absence of travel (31\%) and the use of virtual tools for some activities post-pandemic $(40 \%)$. Conclusion: The use of RSR could complement on-site medical education post-pandemic. More studies should be carried out for a better understanding of the country's reality in the RTR and to advance the discussions in alternative technologies to on-site medical education.
\end{abstract}

Keywords: Medical education, COVID-19, Distance education.

${ }_{1}$ Pontifícia Universidade Católica de Minas Gerais, Betim - MG. *E-mail: victoria.araujo.dias@gmail.com

2 Universidade Federal dos Vales do Jequitinhonha e Mucuri, Diamantina - MG.

3 Universidade Federal do Tocantins, Palmas - TO. 


\section{RESUMEN}

Objetivo: Evaluar la experiencia de los estudiantes de medicina en el Regimén de Enseñaza a Distancia (RED) y su uso pospandémico. Métodos: Se trata de un estudio cualitativo que reporta la experiencia de los estudiantes de medicina en una universidad de Minas Gerais durante el RED. Los estudiantes fueron invitados y recibieron el cuestionario virtualmente y firmaron un consentimiento informado. Resultados: $20 \%$ de los estudiantes de medicina respondieron el cuestionario, predominantemente mujeres, entre 17 y 26 años, que asisten al ciclo clínico. El RER impactó negativamente los estudios del $78,1 \%$ de los encuestados y predominaron los sentimientos de ansiedad (74,8\%) y desánimo (73,5\%). Más del $90 \%$ de los estudiantes faltan a las clases presenciales, la interacción social (48\%) y el entorno académico (45\%). También surgieron puntos positivos, referidos a la rutina del estudio $(35,5 \%)$ y el uso de estas herramientas para algunas actvidades pospandémicas (40\%). Conclusión: El uso de RED podría complementar la educación médica in situ pospandémica. Se deben realizar más estudios para comprender la realidad del país en el RED y avanzar en las discusiones sobre tecnologías alternativas a la educación médica en el sitio.

Palabras clave: Educación médica, COVID-19, Educación a distancia.

\section{INTRODUÇÃO}

Em 2020 o mundo iniciou o enfrentamento à pandemia de COVID-19, desde então várias medidas foram tomadas visando reduzir o número de contaminados e óbitos (BRASIL, 2020). A determinação da suspensão das aulas presenciais nas universidades brasileiras esteve entre essas medidas. No dia 17 de março de 2020, - Ministério da Educação (MEC) por meio da Portaria oㅡ 343 do Diário Oficial da União autorizou, excepcionalmente, a substituição das disciplinas presenciais por aulas que utilizassem meios e tecnologias de informação e comunicação (BRASIL, 2020).

Cada universidade adotou nomenclaturas diferentes, no caso da universidade em que a pesquisa foi realizada tal regime foi nomeado de Regime Letivo Remoto (RLR), cujo objetivo foi viabilizar as atividades acadêmicas e o calendário letivo em uma realidade que demandou distanciamento social. O RLR consistiu na adoção de aulas síncronas, em tempo real, transmitidas por aplicativos próprios da Universidade, nos quais os professores e estudantes foram instruídos a acessar, via internet, as plataformas digitais nos mesmos horários em que as disciplinas iriam ser ministradas presencialmente (BOSKURT A e SHARMA RC, 2020).

Tal medida foi imprescindível para manter o cronograma letivo, porém diversos desafios surgiram com 0 início da sua implementação (BEZERRA IM, 2020). Além dos desafios técnicos e de acessibilidade, também surgiram os desafios humanos, pois estudantes e professores tiveram que se adaptar a uma realidade inédita e atípica e se reinventar frente a essa demanda tecnológica (OLIVEIRA SS et al., 2020).

Várias dúvidas acerca da aplicabilidade e eficácia do RLR no processo de ensino-aprendizagem foram surgindo (BEZERRA IM, 2020). Não se sabia que a implementação do RLR como a pandemia afetou a educação médica global e brasileira e tal assunto ainda não foi esgotado atualmente (MARSILLI LBR, et al., 2020).

Para contribuir ao conhecimento sobre o impacto da substituição das disciplinas presenciais pelos meios e tecnologias de informação e comunicação sobre a educação médica, o presente trabalho objetivou investigar a percepção dos estudantes dentro da comunidade acadêmica de uma universidade privada no Brasil após a implementação do RLR.

\section{MÉTODOS}

O presente estudo foi conduzido por pesquisadores graduandos de medicina em Minas Gerais e Tocantins. Para o presente estudo, os participantes escolhidos foram estudantes de medicina de uma universidade de caráter privado, situada na região metropolitana de Belo Horizonte.

A escolha de tal campo de pesquisa se fundamentou por ser a universidade a qual a maioria dos pesquisadores é vinculada e pela facilidade de acesso à internet da comunidade acadêmica. Tal instituição utilizava o formato de ensino médico tradicional com todas as aulas teóricas ministradas presencialmente. $\mathrm{O}$ 
ensino é baseado na comunidade e centrado no aluno, favorecendo a aquisição de competências, habilidades e atitudes no âmbito do Sistema Único de Saúde desde os primeiros períodos acadêmicos (MINISTÉRIO DA EDUCAÇÃO, 2014).

O currículo preconiza a aquisição de conhecimento em espiral e é dividido em ciclos. Sendo o ciclo básico compreendido nos quatro primeiros semestres e focado na aquisição de conhecimentos fisiológicos. Já o ciclo clínico, que ocorre do quinto ao oitavo período, é focado na aquisição de habilidades semiológicas e o último ciclo, do nono ao décimo segundo período, direcionado aos internatos médicos (MARSILLI LBR, et al., 2020; MINISTÉRIO DA EDUCAÇÃO, 2014).

O estudo conduzido investigou a percepção dos discentes quanto ao RLR durante a pandemia de COVID19 do ano de 2020. A implementação do RLR ocorreu em março de 2020 , em caráter emergencial, com todas as aulas teóricas migradas para ambiente virtual. As aulas que ocorreram de forma síncrona e sendo disponibilizadas de modo assíncrono, através de gravações para que os acadêmicos que não conseguiram comparecer no horário pré-estabelecido pudessem assisti-las. Além disso, as aulas práticas e as atividades em campo estavam suspensas durante o período em que foi feita a pesquisa, inclusive aquelas relacionadas aos internatos médicos.

Os pesquisadores submeteram os protocolos de estudo à apreciação e aprovação pelo Comitê de Ética e Pesquisa da universidade em estudo, sob o número 38161620.7.0000.5137.

Trata-se de um estudo observacional, descritivo, qualitativo, em que foi feito um levantamento por meio de questionário eletrônico (GIL AC, 2002; LAKATOS EM e MARCONI MA, 2003). Este foi constituído de perguntas abertas, dicotômicas e de múltipla escolha. A amostragem do presente estudo se caracteriza por ser não probabilística dos estudantes de medicina em geral (LAKATOS EM e MARCONI MA, 2003).

Após a devida aprovação dos protocolos, todos os acadêmicos de medicina da universidade foram convidados a participarem da pesquisa, por meio de mídias eletrônicas (e-mail e redes sociais). Foi encaminhado em conjunto ao convite de participação um link de acesso contendo o Termo de Consentimento Livre e Esclarecido (TCLE) e um questionário elaborado no Google Forms ${ }^{\circledR}$ composto por perguntas abertas e fechadas. Todos os participantes tiveram acesso ao TCLE antes da pesquisa e somente foram direcionados aos questionários aqueles que completaram sua leitura, concordaram com os termos e receberam cópia por endereço eletrônico do documento assinado pelo pesquisador.

Para melhor delimitar o estudo, a pesquisa teve como critério de inclusão, estudantes de medicina da universidade em questão e, como critério de exclusão, graduandos que estavam com a matrícula trancada durante o período de atividades do RLR, além daqueles envolvidos diretamente com a pesquisa. Um mecanismo de validação para que cada estudante pudesse participar uma única vez da pesquisa foi criado no próprio formulário. Foi assegurado anonimato. Nenhum pesquisador teve acesso à identificação dos pesquisados.

Para respostas obtidas por perguntas fechadas, o próprio Google Forms ${ }^{\circledR}$ realizou o agrupamento e apresentou gráficos. Já para as respostas abertas, estas foram analisadas por grupos de três pesquisadores cada. Respostas semelhantes foram agrupadas qualitativamente em categorias para embasar os resultados da pesquisa e as conclusões obtidas (LUDKE M e ANDRÉ EDAM, 2018). O uso de categorização qualitativa das questões abertas e posterior revisão destas pelos demais pesquisadores permitiu melhor fluidez da análise dos dados captados, uma vez que muitos estudantes apontaram vários fatores pesquisáveis em suas respostas abertas, que já haviam apresentado em respostas fechadas. Como um respondente pode apresentar mais de um item à uma resposta aberta, os dados apontados apresentam somatória superior a $100 \%$ dentre os itens categorizados.

\section{RESULTADOS}

Ao todo 155 , aproximadamente $20 \%$ dos 776 estudantes regularmente matriculados à época no curso de medicina da universidade responderam a esta pesquisa. O perfil predominante dos participantes consistiu em mulheres com idade entre 17 e 26 anos. A maioria dos respondentes estava matriculada em algum semestre inserido no ciclo clínico $(51,6 \%)$. Os autores supõem que a participação majoritária de acadêmicos de tal ciclo 
pode ser justificada pela suspensão das atividades em campo e das aulas práticas, causando maior prejuízo para essa parcela de estudantes, o que os motivaria a participar de tal inquérito. Além disso, a maioria dos estudantes responderam estar matriculados em sete a oito disciplinas $(43,9 \%)$ transmitidas em meio virtual durante o RLR. Os dados acima e outros obtidos pela parte objetiva do questionário estão compilados na Tabela 1.

Tabela 1 - Perfil dos estudantes respondentes.

\begin{tabular}{lc}
\hline Variáveis & $\mathbf{N}(\%)$ \\
\hline Sexo & $122(78,7 \%)$ \\
\hline Feminino & $33(21,3 \%)$ \\
Masculino & $112(72,2 \%)$ \\
\hline Idade & $33(21,3 \%)$ \\
\hline $17-25$ & $10(6,5 \%)$ \\
$26-35$ & \\
$\geq 36$ & $49(31,6 \%)$ \\
\hline Período do curso & $80(51,6 \%)$ \\
\hline Ciclo básico (10 ao 40 período) & $22(14,3 \%)$ \\
Ciclo clínico (50 ao 8o período) & $4(2,6 \%)$ \\
Internato (9o ao 120 período) & \\
Irregulares & $29(18,7 \%)$ \\
\hline Matriculado em & $123(79,4 \%)$ \\
\hline $1-5$ disciplinas & $3(1,9 \%)$ \\
$6-10$ disciplinas & \\
$11-14$ disciplinas & $30(19,4 \%)$ \\
\hline Disciplinas por EAD & $17(11 \%)$ \\
\hline $1-2$ & $28(18,1 \%)$ \\
$3-4$ & $68(43,9 \%)$ \\
$5-6$ & $10(6,5 \%)$ \\
$7-8$ & $2(1,3 \%)$ \\
$9-10$ & \\
$\geq 11$ & $154(99,4 \%)$ \\
\hline Acesso à internet & $1(0,6 \%)$ \\
\hline Sim, no domićlio & \\
Sim, fora do domicílio & $91(58,7 \%)$ \\
\hline Qualidade do acesso à internet & $58(37,4 \%)$ \\
\hline Bom & $6(3,9 \%)$ \\
Razoável & \\
Ruim & \\
\hline
\end{tabular}

Fonte: Freire MM, et al. 2021.

Além dos resultados citados anteriormente, a Tabela 1 apresenta que 99,4\% dos participantes responderam que possuíam acesso à internet em casa e apenas um acadêmico referiu que precisava sair de casa para obter acesso.

Em relação a qualidade de acesso, 58,7\% o classificaram como bom, 32,4\% como razoável e 3,9\% como ruim. Em torno de $74 \%$ dos estudantes relataram que experimentaram momentos em que a internet apresentou alguma falha durante a aula online. Quanto ao acesso aos livros digitais como forma de estudo, apenas $46,7 \%$ dos participantes da pesquisa disseram estar muito satisfeitos.

Esta pesquisa deu enfoque ao aspecto qualitativo da experiência do discente e abordou os sentimentos mais presentes durante o período de regime remoto. Para as perguntas de cunho qualitativo os estudantes poderiam marcar mais de uma das opções disponíveis, portanto o somatório das respostas extrapola 100\%.

Os sentimentos predominantemente marcados pelos participantes foram: ansiedade $(74,8 \%)$, desestímulo $(73,5 \%)$, estresse $(67,1 \%)$ e angústia $(47,7 \%)$. Outros sentimentos pontuados foram: conforto $(32,2 \%)$, tranquilidade (11\%), satisfação $(5,8 \%)$ e motivação $(4,5 \%)$. Dentro do próprio questionário, os estudantes poderiam relatar através de texto corrido seus sentimentos quanto ao RLR. As respostas foram então categorizadas e compiladas no Gráfico 1. 
Gráfico 1 - Sentimentos gerais apontados pelos estudantes pesquisados predominantes no Regime Letivo Remoto.

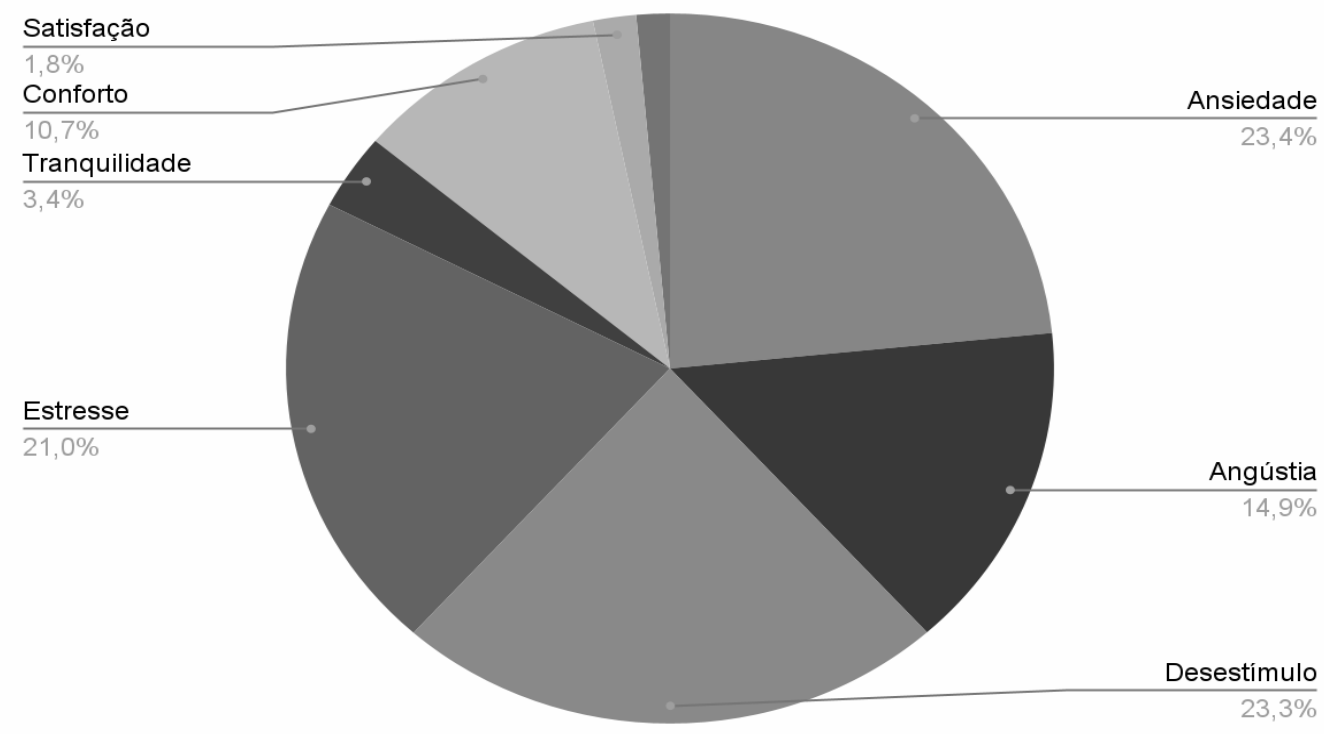

Fonte: Freire MM, et al. 2021.

Através de perguntas abertas os participantes foram estimulados a comparar os seus sentimentos quanto ao RLR com o formato presencial: $92,3 \%$ dos estudantes responderam que sentiam falta das aulas presenciais, principalmente da interação social com outros colegas e professores (48\%), da ambientação promovida pela faculdade $(23,6 \%)$, da capacidade de concentração $(11,6 \%)$, das disciplinas práticas $(11,6 \%)$, da rotina e disciplina (9,5\%) e do estímulo de estudo (3,7\%). O Gráfico 2 elenca tais categorias de respostas.

Gráfico 2 - RLR x Presencial: O que mais faz falta para os estudantes de medicina.

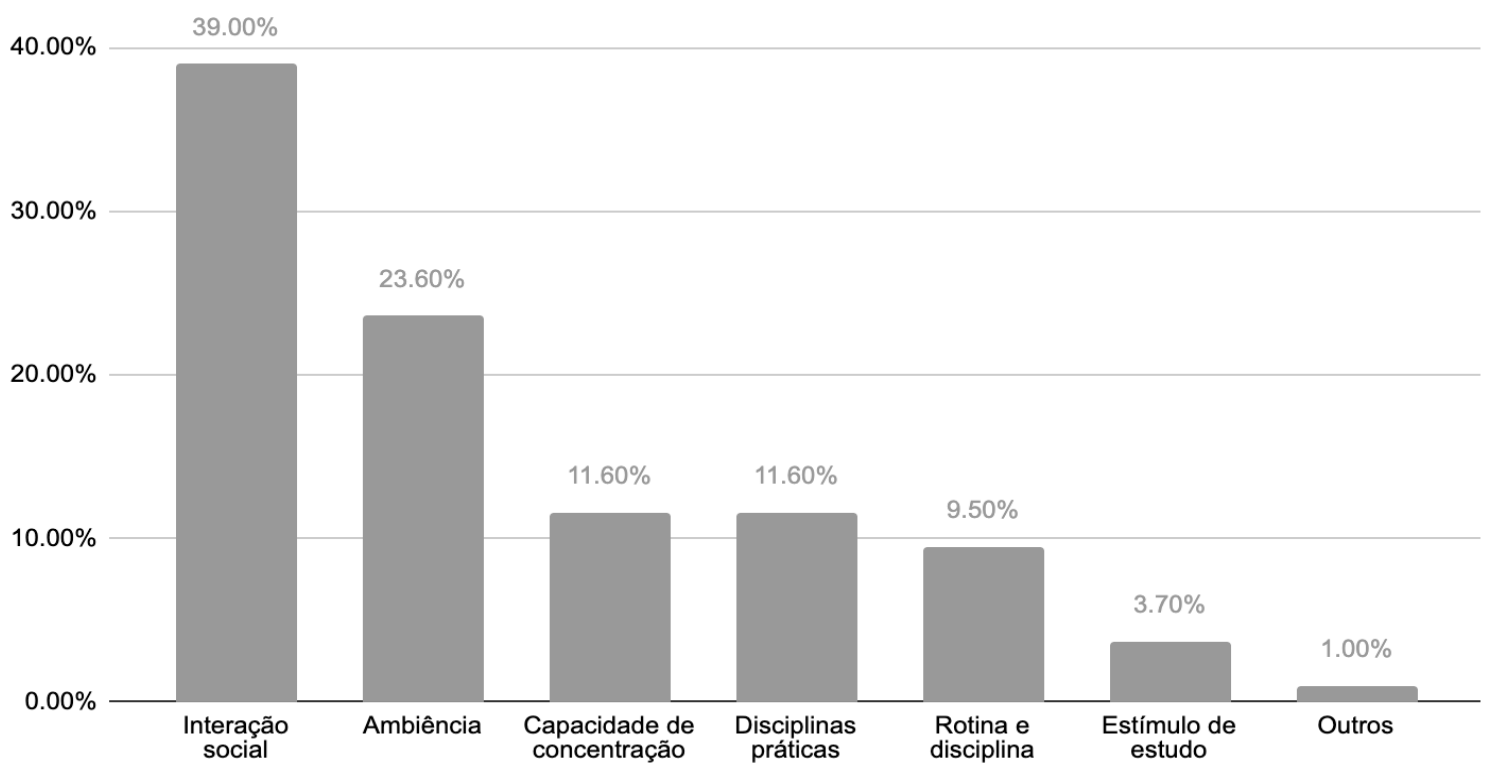

Fonte: Freire MM, et al. 2021.

Os alunos puderam citar fatores de impacto positivo ou negativo do RLR, $78,1 \%$ afirmaram que o ensino remoto impactou negativamente em sua rotina de estudos. Cerca de metade dos respondentes relatou desconforto em participar ativamente das aulas, bem como se sentiram desconcentrados no ambiente domiciliar. $67,1 \%$ dos estudantes responderam que o estudo online prejudicou sua ergonomia e a diminuição no desempenho do aprendizado foi relatada por $68,4 \%$ dos participantes. 
Ainda neste quesito os estudantes puderam expressar quais fatores impactam negativamente seu processo de aprendizagem no RLR. As respostas foram categorizadas qualitativamente e apresentadas no Gráfico 3: decaimento da qualidade de ensino (25.2\%), fatores emocionais (17\%), redução da interação social $(16,3 \%)$, disciplina e concentração (15\%), exaustão por tempo de tela $(11,6 \%)$ e acesso à internet $(8,9 \%)$.

Gráfico 3 - Fatores de impacto negativo do RLR, comparado ao ensino presencial, no processo de aprendizagem dos estudantes.

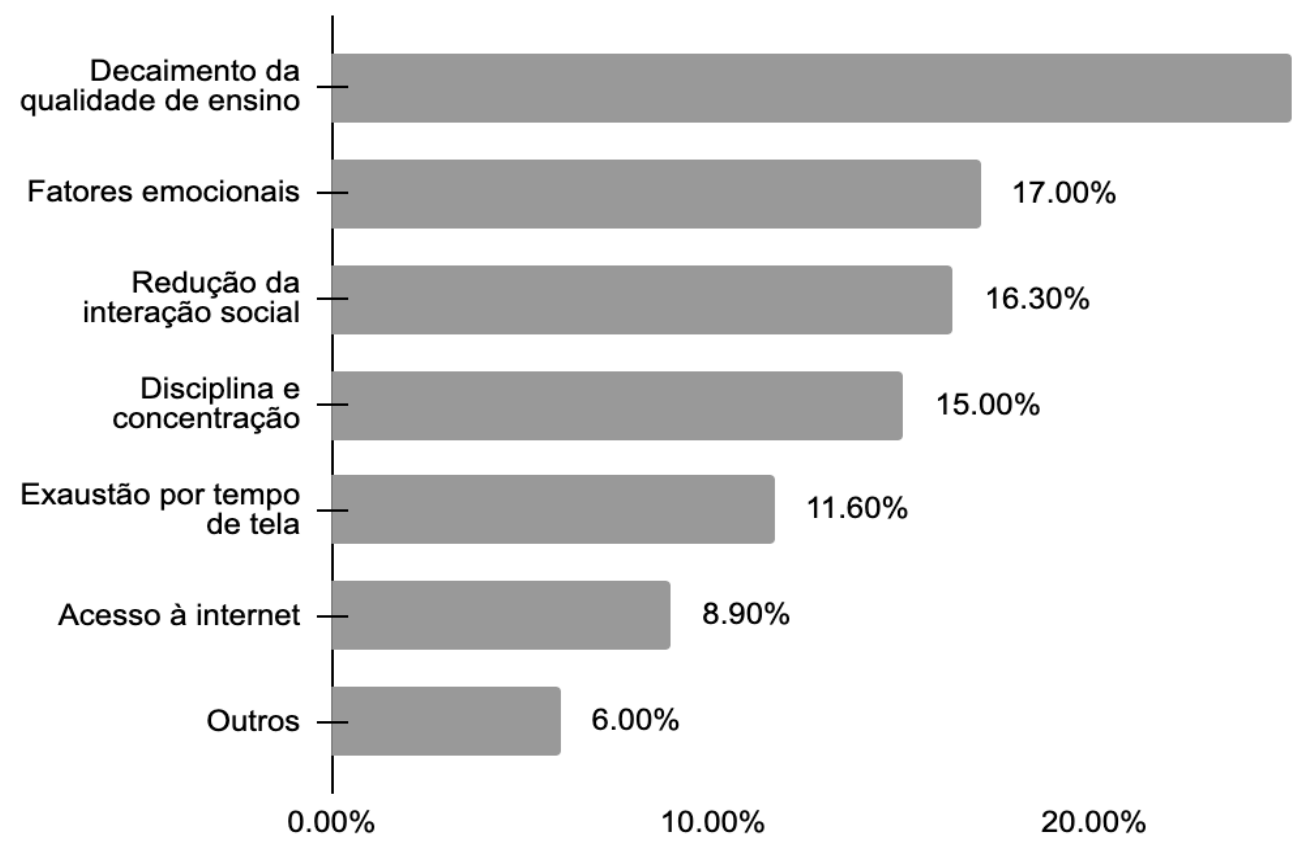

$25.20 \%$

Fonte: Freire MM, et al. 2021.

Já os principais pontos positivos do RLR elencados pelos estudantes foram: estabelecimento de uma rotina de estudos (35,5\%), otimização do tempo por não precisar se deslocar até o local da faculdade (31\%), conforto e comodidade (22\%), bem como, o fator financeiro (6,5\%), conforme apresentado no Gráfico 4.

Gráfico 4 - Fatores de impacto positivo do RLR, comparado ao ensino presencial, no processo de aprendizagem dos estudantes.

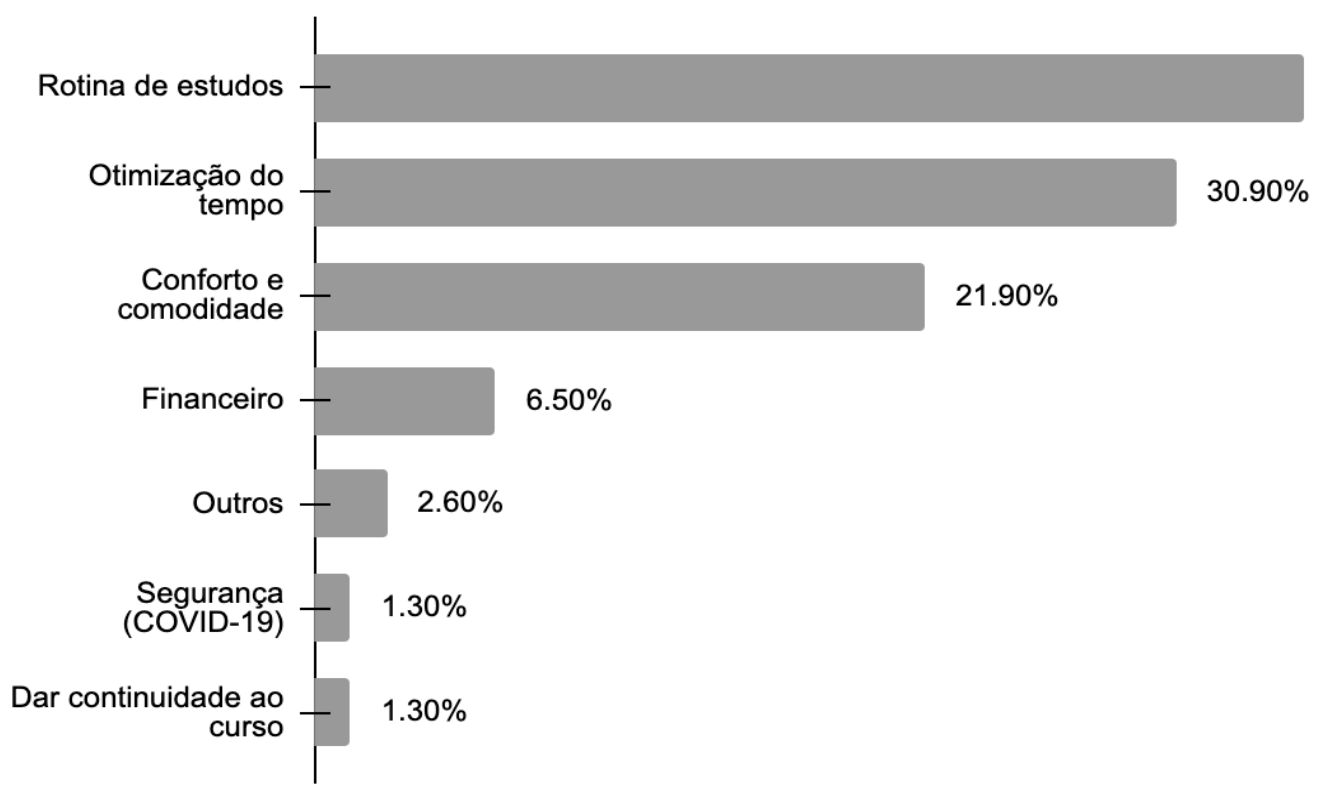

Fonte: Freire MM, et al. 2021. 
Enfim, quando questionados em relação a continuidade do RLR após a pandemia, 40\% dos estudantes concordaram com a utilização dessa ferramenta para algumas matérias e reuniões e $29 \%$ apenas para reuniões de mentoria, ligas acadêmicas e trabalhos científicos. Já $28,4 \%$ dos acadêmicos afirmaram que preferem todas as atividades universitárias no formato presencial.

\section{DISCUSSÃO}

A introdução do RLR, no curso de medicina, trouxe diversos questionamentos dos acadêmicos sobre a efetividade dessa forma de ensino em comparação ao modo presencial (BEZERRA IM, 2020). Alguns pontos sobre este tema endossam preocupação com a qualidade do ensino nesta alternativa metodológica durante o distanciamento social imposto pela pandemia de COVID-19: falta de habilidades e de treinamentos para que estudantes e professores consigam lidar com o ambiente virtual; engajamento nas aulas virtuais; problemas técnicos; manejo do tempo e falta de assistência por parte das instituições de ensino (PEI LU E WU H, 2019; O'DOHERTY D, et al., 2018).

O perfil socioeconômico interfere no acesso a um computador com internet e na implementação do RLR (JAMUR HR e BRITO GIS, 2017). Dados gerais de inclusão digital abordados na Pesquisa TIC Domicílios 2019 evidenciam que $71 \%$ dos domicílios brasileiros possuem acesso à internet. Porém, existem desigualdades entre as classes sociais, pois somente $50 \%$ da população das classes $D$ e $E$ possui tal acesso, confrontando com 99\% e 95\%, respectivamente, das pessoas das classes A e B. Portanto, a dificuldade de inclusão digital pode ser mínima frente a uma situação socioeconômica mais favorável presente em acadêmicos de universidades privadas (CENTRO REGIONAL DE ESTUDOS PARA O DESENVOLVIMENTO DA SOCIEDADE DE INFORMAÇÃO- CETIC, 2020).

Apesar de considerarmos um viés metodológico em nosso estudo uma vez que somente aqueles que tiveram acesso à internet poderiam responder tal questionário, esta era a única forma exequível de aplicação do questionário frente ao distanciamento social.

Nossa pesquisa apontou que $99,4 \%$ dos estudantes participantes possuíam acesso à internet em casa, concordando com dados da Pesquisa TIC Domicílios 2019, o que facilitaria a adoção do RLR (CETIC, 2020). Ainda assim, neste cenário de grande inclusão digital, 37,4\% dos participantes classificaram a velocidade de acesso à internet como razoável e 16,1\% referiram que a internet parou de funcionar durante a transmissão da aula virtual. Apesar da grande maioria apontar facilidade com ferramentas virtuais, $38,6 \%$ relataram pouco e moderado conhecimento em informática, o que poderia comprometer o aprendizado por meio das plataformas digitais.

Outro ponto importante evidenciado pelo presente trabalho foi o isolamento social e a consequente transição do ambiente físico para o virtual. Uma pesquisa realizada mensurou que mais de $75 \%$ dos entrevistados não sabiam como se estruturava o RLR (ALSOUFI A, et al, 2020). Tal desconhecimento, tanto pelo corpo docente e quanto pelo discente, gerou inúmeros desafios durante a implementação da modalidade nas universidades de todo o mundo. Tal transição foi acompanhada pela redução da performance acadêmica, que também foi evidenciado na literatura (ROSE S, 2020; MEO SA, et al., 2020). Não é de se estranhar que a maioria dos estudantes respondeu que sente falta de aulas presenciais $(92,3 \%)$, dado que é observado em pesquisas anteriores (PEREIRA SRB e SANTOS GF, 2019; CAPELETTI AM, 2014).

O fator negativo frente ao RLR mais apontado pelos acadêmicos em nossa pesquisa foi a falta de interação com colegas e professores (48\%), seguido pelo sentimento de falta do ambiente acadêmico como um todo: $45 \%$ disseram sentir falta da sala de aula, da biblioteca e do ambiente universitário e do espaço físico em si.

Nesse sentido, é importante frisar que a sala de aula, o ambiente da universidade e o convívio com colegas proporcionam incentivo para o processo de aprendizagem e para a construção de uma rotina. Na ausência do ambiente acadêmico, os estudantes são mais propícios a ter estratégias de aprendizado ineficazes, baixa motivação e engajamento e comunicação reduzida. Isso pode se dever a falta de foco, interesse em outras atividades do ambiente doméstico e dificuldades técnicas (MEO SA, et al., 2020; WILCHA RJ, 2020). No presente estudo, $15 \%$ dos estudantes citaram a falta de rotina e disciplina como um ponto negativo do RLR, influenciando diretamente nos seus estudos e aproveitamento das disciplinas. 
Um dos motivos para a diminuição do engajamento discente é a perda da relação entre estudantes e professores. A realização de aulas por plataformas online dificulta a identificação das reações dos estudantes e possibilita manter uma postura mais passiva frente ao processo de ensino-aprendizado (WILCHA RJ, 2020; OLIVEIRA MF e ARAUJO LMB, 2019). Durante uma aula, o feedback constante é importante para que o professor possa ajustar a didática e manter o interesse dos estudantes; o contato não-verbal e visual neste ponto essencial (WILCHA RJ, 2020, O'DOHERTY D, et al., 2018; ROBERTS V, et al., 2020).

Além das dificuldades de engajamento às aulas virtuais, as distrações do domicílio exigem dos estudantes mais disciplina e autonomia (ROBERTS V, et al., 2020). Esse fato foi corroborado por nosso estudo, no qual $26,5 \%$ dos participantes acreditaram que o ambiente domiciliar influenciou negativamente na concentração e desempenho durante as aulas de transmissão virtual. Uma grande preocupação é a falta de motivação, baixo engajamento nas aulas e um ambiente que propicia perda de foco, que somados podem ter grande impacto na aprendizagem dos acadêmicos e requer discussões. Há que se pensar em maneiras de melhorar a didática nas aulas virtuais, mas também é função das universidades promover estratégias para melhorar esse cenário (PEREIRA SRB e SANTOS GF, 2019; ASHOKKA B, et al., 2020).

Nossos resultados indicaram que $25 \%$ dos entrevistados consideraram que a qualidade de ensino durante o RLR caiu drasticamente, aumentando a preocupação com seu futuro acadêmico e profissional. Em associação, quase $12 \%$ dos respondentes revelaram que a ausência de aulas práticas contribui bastante para piora da qualidade de ensino. Esse problema também foi discutido em uma pesquisa realizada com acadêmicos ingleses, na qual mais de $75 \%$ dos entrevistados consideraram o ensino virtual como não efetivo e mais de $80 \%$ sentiram que as competências clínicas não foram desenvolvidas corretamente (DOST S, et al., 2020). Outro estudo apontou que quase $70 \%$ dos entrevistados declararam que não gostaram do RLR e mais de 60\% acharam que aprenderam menos nessa nova realidade (BROTONS P, et al., 2020).

Apesar dos pontos negativos do ambiente virtual, plataformas online de estudos oferecem comodidades como não ter limitações de horário ou local para assistirem as aulas e proporcionar um acesso mais amplo e mais rápido a diferentes conteúdos (GORDON M, et al., 2020; PEI L e WU H, 2019; O'DOHERTY D, et al., 2018; WILCHA RJ, 2020). O RLR foi apontado como positivo e negativo na rotina de estudos dos nossos pesquisados: $35,5 \%$ consideraram o ensino remoto como benéfico para organização e planejamento dos estudos, enquanto $15 \%$ referiram diminuição da disciplina e da concentração.

Tal benefício de flexibilidade do ensino remoto já era conhecido desde antes da pandemia, tanto na graduação, quanto em cursos complementares. Em alguns relatos, os estudantes preferiram assistir às gravações das aulas do que estarem presentes nas videoconferências online ( $P E I L$ e WU H, 2019; O'DOHERTY D, et al., 2018; ROBERTS V, et al., 2020; HILBURG R, et al., 2020). 40\% dos nossos pesquisados concordaram em dar continuidade na utilização das ferramentas virtuais para algumas disciplinas e reuniões após a pandemia.

Outra temática abordada pelo questionário do presente estudo foi o prejuízo na saúde mental dos acadêmicos de medicina: $74,8 \%$ relataram ansiedade, $73,5 \%$ desestímulo e $67,1 \%$ estresse. Ademais, os fatores emocionais $(16,1 \%)$ foram o segundo aspecto de maior preocupação entre os estudantes entre os pontos negativos do RLR. Tais dados corroboram com outras pesquisas encontradas na literatura, que também apontam danos na saúde mental e a presença de sintomas depressivos e de ansiedade nos estudantes de medicina (ALSOUFI A, et al., 2020; LYONS Z, et al., 2020). Isso mostra que a pandemia e o RLR contribuíram para o agravamento da saúde mental dos estudantes de medicina, que já era comprometida anteriormente, com alta taxa de depressão, ideação suicida e de Síndrome de Burnout entre os acadêmicos (OLIVEIRA MF e ARAUJO LMB, 2019).

Durante o RLR há também a necessidade de que os estudantes possuam condições ergonômicas favoráveis e evitem doenças osteomusculares. Uma pesquisa revelou que nas atuais condições do Brasil, $8 \%$ dos discentes não possuem sequer mesas e cadeiras apropriadas para as aulas virtuais, tornando as condições ergonômicas totalmente inadequadas. Nesse mesmo estudo, $53,8 \%$ dos participantes possuem pelo menos uma cadeira adequada, mas sem espaço físico preparado. Já $38,2 \%$ relataram ter condições 
totalmente favoráveis, com um escritório com mobílias confortáveis e adequadas (BRIDI MA, et al., 2020; SAGÁT P, et al., 2020). Esses dados complementam nossos achados na presente pesquisa, uma vez que $67,1 \%$ dos estudantes responderam que não se sentem ergonomicamente confortáveis durante o RLR, contra $32,9 \%$ que relataram o contrário.

Nossa pesquisa convidou os estudantes a refletirem sobre a continuidade do RLR após a pandemia. Quase $30 \%$ dos entrevistados preferiram que todas as atividades acadêmicas fossem presenciais e cerca de $40 \%$ gostariam da continuidade do RLR para algumas matérias e reuniões. Tais achados corroboram com recente estudo multicêntrico brasileiro, em que mais da metade dos acadêmicos (52,3\% na rede privada e $51,3 \%$ na pública) preferem continuar com as aulas totalmente presenciais após a pandemia e $21,7 \%$ gostariam que o curso fosse oferecido de forma híbrida (parte presencial e parte online) (OLIVEIRA A, et al., 2021).

\section{CONCLUSÃO}

A partir do estudo e da revisão da literatura realizados, foi observado que a substituição de aulas presenciais em emergencial no ensino superior afetou diretamente os estudantes de medicina. A percepção negativa foi preponderante nesta vivência, mas o RLR também se apresentou benéfico em quesitos relacionados à flexibilidade e praticidade. Os autores acreditam que o presente estudo contribui ao conhecimento científico em educação médica ao levantar primariamente os sentimentos apontados pelos acadêmicos de medicina frente ao RLR. Considerando a relevância do tema, estudos similares devem ser estimulados em outras instituições, para que se possa avançar em discussões sobre tecnologias alternativas ao ensino médico presencial em forma de contingência.

\section{REFERÊNCIAS}

1. ALSOUFI A, et al. Impact of the COVID-19 pandemic on medical education: Medical students' knowledge, attitudes, and practices regarding electronic learning. PLoS One, 2020; 15(11): 1-20.

2. ASHOKKA B, et al. Coordinated responses of academic medical centres to pandemics: Sustaining medical education during COVID-19. Medical Teacher, 2020; 42(7): 1-10.

3. BEZERRA IM. State of the art of nursing education and the challenges to use remote technologies in the time of Corona Virus Pandemic. Journal of Human Growth and Development, 2020; 30(1): 141-7.

4. BOSKURT A, SHARMA RC. Emergency remote teaching in a time of global crisis due to CoronaVirus pandemic. Asian Journal of Distance Education, 2020; 15(1): 1-6.

5. BRASIL. Atos do Poder Executivo. Medida Provisória no 927, de 22 de março de 2020. Dispõe sobre as medidas trabalhistas para enfrentamento do estado de calamidade pública reconhecido pelo Decreto Legislativo ํㅡ 6 , de 20 de março de 2020, e da emergência de saúde pública de importância internacional decorrente do coronavírus (covid-19), e dá outras providências. Diário Oficial da União, ed. 55-L, Brasília-DF, 2020a. Disponível em: https://www.in.gov.br/en/web/dou/-/medida-provisoria-n-927-de-22-de-marco-de-2020-249098775. Acesso em: 3 set. 2021.

6. BRONTONS P, et al. Perceptions of students confined by the COVID-19 pandemic on distance learning in medicine. Rev. méd. Chile [Internet], 2020; 148(10):1461-1466.

7. CAPELETTI AM. Ensino a distância: Desafios encontrados por alunos do ensino superior. Saberes da Educação, 2014; 5(1):1-10.

8. CENTRO REGIONAL DE ESTUDOS PARA O DESENVOLVIMENTO DA SOCIEDADE DA INFORMAÇÃO (CETIC). TIC Domicílios 2019.

Disponível

em:

<https://www.cetic.br/media/analises/tic_domicilios_2019_coletiva_imprensa.pdf>. Acesso em: 3 set. 2021.

9. DOST S, et al. Perceptions of medical students towards online teaching during the COVID-19 pandemic: A National Cross-sectional Survey of 2721 UK Medical Students. BMJ Open, 2020; 1-10.

10. GIL AC. Como elaborar projetos de pesquisa. 4nd ed. São Paulo: Atlas, 2002; 175p. GORDON M, et al. Developments in medical education in response to the COVID-19 pandemic: A rapid BEME systematic review: BEME, 2020; 63: 1202-1215.

11. HILBURG R, et al. Medical Education During the Coronavirus Disease-2019 Pandemic: Learning From a Distance. Adv Chronic Kidney Dis, 2020; 27 (5): 412-417.

12. JAMUR HR, BRITO GIS. Uso do material didático na EaD do ponto de vista dos estudantes. Dissertação (Mestrado em Educação) - Biblioteca de Ciências Humanas e Educação. Universidade Federal do Paraná, Curitiba, 2017; 103p. 
13. LAKATOS EM, MARCONI MA. Fundamentos de metodologia científica. 5nd ed. São Paulo: Atlas, $2003 ; 311 \mathrm{p}$.

14. LUDKE M, ANDRÉ EDAM. Pesquisa em educação: abordagens qualitativas. 2nd ed. Rio de Janeiro: Anthares, 2018, $102 p$.

15. LYONS Z, et al. COVID-19 and the mental well-being of Australian medical students: Impact, concerns and coping strategies used. Australas Psychiatry, 2020; 28(6): 649-652.

16. MARSILLI LRB, et al. Ensino médico na pandemia de COVID-19: ponto de vista de acadêmicos de medicina. Medicina (Ribeirão Preto), 2020; 53(4): 490-494.

17. MEO SA, et al. COVID-19 Pandemic: impact of quarantine on medical students $\because$ mental wellbeing and learning behaviors. Pakistan Journal Of Medical Sciences, 2020; 36(19):43-48.

18. MINISTÉRIO DA EDUCAÇÃO. Resolução CNE/CES n 3, de 20 de junho de 2014 - Institui Diretrizes Curriculares Nacionais do Curso de Graduação em Medicina e dá outras providências. Disponível em:http://portal.mec.gov.br/escola-de-gestores-da-educacao-basica/323-secretarias-11287 7938/orgaos-vinculados-82187207/20138-ces-2014. Acesso em: 28 set. 2021.

19. O'DOHERTY D, et al. Barriers and solutions to online learning in medical education: An integrative review. BMC Medical Education. 2018; 18(1):1-11.

20. OLIVEIRA A, et al. COVID-19 e Educação: Os impactos no ensino superior a partir dos enfrentamentos, potencialidades e perspectivas futuras. Disponível em: https://www.eventoanap.org.br/data/inscricoes/8805/form4428251888.pdf. Acesso em: 3 set. 2021.

21. OLIVEIRA MF, ARAUJO LMB. Saúde Mental Do Estudante de Medicina. Brazilian Journal of Development, $2019 ; 5$ (11): 23440-52.

22. OLIVEIRA SS, et al. As Escolas Médicas e os desafios da formação médica diante da epidemia brasileira da COVID19: das (in)certezas acadêmicas ao compromisso social. Aps em Revista, 2020; 2(1):56-60.

23. PEI L, WU H. Does online learning work better than offline learning in undergraduate medical education?: A systematic review and meta-analysis. Medical Education Online, 2019; 24(1):1-14.

24. PEREIRA SRB, SANTOS GF. Um olhar crítico sobre a qualidade do acesso à internet, na interatividade e a qualidade de aprendizagem. Rev Humanidades e Inovação, 2019; 6(9):202-211.

25. ROBERTS V, et al. Peer teaching medical students during a pandemic. Medical Education Online, 2020; 25(1):1772014.

26. ROSE S. Medical Student Education in the Time of COVID-19. JAMA, 2020; 323 (21):2131-2132.

27. SAGÁT P, et al. Impact of COVID-19 Quarantine on Low Back Pain Intensity, Prevalence, and Associated Risk Factors among Adult Citizens Residing in Riyadh (Saudi Arabia): a cross-sectional study. International Journal Of Environmental Research And Public Health, 2020; 17(19).

28. TOKUÇ B, VAROL G. Medical Education in Turkey in the Time of COVID-19. Balkan Medical Journal, 2020; 37(4): 180-181.

29. BRIDI MA, et al. Relatório técnico-científico da pesquisa: o trabalho remoto/home office no contexto da pandemia Covid-19. Curitiba: Universidade Federal do Paraná, Grupo de Estudos Trabalho e Sociedade, 2020; 79 p.

30. WILCHA RJ. Effectiveness of Virtual Medical Teaching During the COVID-19 Crisis: Systematic Review. JMIR Med Educ, 2020; 6(2). 